\title{
A dependency syntax of German
}

\author{
Henning Lobin \\ Bonn, Federal Republic of Germany
}

\section{The German language: some general remarks}

German is an inflected language with a rich morphology for derivation purposes. Nouns and adjectives derived from verbs with an extensive valency can function as the heads of very extensive phrases. In phrases of this kind, a lot of dependents precede the head. The derivation of the valency of such nouns or adjectives can be described in general by the use of valency transformations.

For German syntax, the word order is a typical issue, in particular, the position of the elements within the verbal complex (VC). If possible, the VC occurs in two parts or as one part of a two part frame:

- finite part in the second position, remainder of the VC in the last position (declarative clause word order)

- finite part in the first position, remainder of the VC in the last position (interrogative clause word order)

- imperative verb in the first postion (imperative clause word order)

- subjunction in the first position, VC in the last position (subordinate clause word order)

Every German clause contains such a "clause frame", but in some cases the frame is 
inherent in the clause (if the VC is a finite verb in the present or past tense). The clause frame appears, if the VC is transformed into another tense or into a modal verbal complex. The elements inside the clause frame are subject to several other constraints such as, for instance, that pronominal complements usually precede all lexical complements.

\section{Word classes}

Verb (V). Verbs are the words which can occur in a finite form or as participle II. Sample words in the infinitive form: gehen, lachen, trinken, gewinnen, sein, werden, haben, scheinen, helfen, lassen, können, dürfen; participle II, verbs like gekommen or vergnügt, are Vs just in case they function as a CVRB dependent.

Noun (N). Nouns are the words which always occur in the same gender. Sample words in the nominative singular: Mann, Haus, Wohnung, Selbstverständlichkeit, Maschine, Gebirge, Laufen.

Determiner (Det). Determiners are the words which commute with a proper noun in the genitive when placed in front of a noun (Oskars Vater - mein Vater). Sample words in the nominative singular: der, die, das; dieser, diese, dieses; mein, dein, sein; all(e); viel; welcher, welche, welches; kein, keine; neunzig (cardinal number); also the Dets, which introduce a relative clause: dessen, deren.

Adjective (A). Adjectives are the words, which can occur between a determiner and a noun. Sample words (all in the nominative singular masculine): gro $\beta$, schnell, hiesig, behördlich, Hamburger (or hamburger), eisern, laufend (participle I), vergnügt (participle II, not in all cases an A), zweite (ordinal number).

Pronoun (Pro). Pronouns are the words, which commute with a noun phrase. Sample words (only in nominative singular): ich, du, der, dieses, niemand, etwas, nichts, wer (interrogative pronoun).

Preposition (P). Prepositions are the words, which are invariable and which can occur in front of a noun at any time. Sample words: von, bei, an, auf, bis, mithilfe, vermöge, zeit.

Subjunction (Sbj). Subjunctions are the words, which have the function to make clauses subordinate to other words. Sample words: $d a \beta, o b$, weil, nachdem, zu, obwohl, indem.

Adverb (Adv). Adverbs are the words, which can occur in the first position within a clause and which can act as an answer to a wh-question. Sample words: jetzt, hier, immer, aufwärts, rechtens, einmal; darin, darüber (prepositional adverbs); wann, worüber (adverbial interrogatives); gern, lieber, (am) liebsten (comparative adverbs).

Conjunction (Con). Conjunctions are the invariable words which link homogeneous and equivalent constructions, whereby the linked word is not changed. Sample words: aber, und, oder, denn (links only VPs), sowie (links only NPs, ProPs, and DetPs), "," (comma syndesis). 
Particle (Par). Particles are all other invariable words. Sample words: möglicherweise, vielleicht, wohl, eigentlich, ja, erstens, wo, als, wie, sehr.

In the following, "VP", "NP" and "DetP" mean verb phrase, noun phrase, determiner phrase and so on for all other word classes.

\section{Short definition of the dependent types}

In this grammar, the first step taken to distinguish complements from adjuncts is to ask whether or not a phrase is forced to occur with a certain word. All obligatory phrases are complements. Therefore, an optional phrase is a complement, if it only occurs with elements of a subclass from within a certain word class. All remaining phrases are adjuncts.

\subsection{Complements}

Subject (CSUB) in a narrow sense; cf. 3.3. All complements which are interchangeable with a personal pronoun in the nominative and which are always governed by a V.

Expletivum and suppletivum (CSUE). Different kinds of $e s$, which are not interchangeable with any other phrase and which are always governed by a V.

Accusative complement (CACC). All complements, which are interchangeable with a personal pronoun in the accusative.

Dative complement (CDAT). All complements, which are interchangeable with a personal pronoun in the dative.

Genitive complement (CGEN). All complements, which are interchangeable with dessen oder deren (i.e. a demonstrative pronoun in the genitive).

Prepositional complement (CPRE). All complements, which are interchangeable with $d a$ - plus a specific $P$.

Situative complement (CSIT). All complements, which are interchangeable with place adverbs like $d a$ or dort and which always occur obligatorily.

Directive complement (CDIR). All complements which are interchangeable with directional adverbs like dahin or daher and which always occur obligatorily.

Expansive complement (CEXP). All complements, which are interchangeable with (um) soviel, -lange, -weit etc. (expansive adverbs or PPs with um).

Nominal complement (CNOU). All complements which, if governed by a V, are interchangeable with es and also occur obligatorily. CNOU can always be expressed by an NP. For other governors see the tables below. 
Adjectival complement (CADJ). All complements which are interchangeable with so and which occur obligatorily. CADJ is always expressed by an AP or by a phrase which is interchangeable with an AP.

Verbal complement (CVRB). All complements which are interchangeable with es geschehen, es tun, es sein etc. CVRB is always expressed by a VP.

Subordinate complement (CSBJ). All complements which are always expressed by a SbjP.

Particle complement (CPAR). All complements which are always expressed by a ParP.

Comparative complement (CCOM). All complements which are always governed by the comparative morpheme of an Adj or Adv.

Reflexive complement (CREF). All obligatory ProPs which are always expressed by a reflexive pronoun (mich, dich, sich).

Prefix complement (CPRX). Only verbal prefixes, which are divided from the verb, if it occurs in the first or the second position of the clause.

\subsection{Adjuncts}

Verbal adjunct (AVRB). All VP adjuncts.

Nominal adjunct (ANOU). All NP and ProP adjuncts which are not AAPPs, and all DetPs which are not governed by an $\mathrm{N}$.

Determinal adjunct (ADET). All DetP adjuncts which are governed by an $\mathrm{N}$.

Adjectival adjunct (AADJ). All AP adjuncts which are not AAPPs.

Prepositional adjunct (APRE). All PP adjuncts, which are not AAPPs.

Subjunctional adjunct (ASBJ). All SbjP adjuncts.

Adverbial adjunct (AADV). All AdvP adjuncts.

Particle adjunct (APAR). All ParP adjuncts.

Apposition (AAPP). An adjunct to $\mathrm{N}$ or Pro which always follows its governor and which is always expressed by an NP, AP or PP.

\subsection{Valency transformations}

For a complete dependency syntax, a description of some fundamental valency transformations must be given. For instance, the subject can occur not only in the nominative case, but also in the accusative:

Peter läßt uns kommen.

Verbs like lassen force the CSUB of kommen to be transformed into a phrase in the 
accusative. The correct name for CSUB must be, strictly speaking, CNOM (Nominative complement), and the subject ought to be a category that can be expressed by CNOM or CACC, and CNOM and CACC ought in turn to be a category which can be expressed as VPs, NPs, DetPs etc. But up to now, these kinds of transformations are not very well developed.

\section{Explicit definition of phrases and dependent types}

Each of the following subsections illustrates the government capacity of a certain word class with an overview table and sample sentences. In the sentences, the governor of the illustrated relation is given in italics and the dependent in bold face.

\subsection{Dependents of verbs}

\begin{tabular}{|c|c|c|c|c|c|c|c|c|c|c|}
\hline & $\mathbf{V}$ & $\mathbf{N}$ & Det & A & Pro & P & Sbj & Adv & Par & Con \\
\hline \hline CSUB & $\times$ & $\times$ & $\times$ & & $\times$ & & $\times$ & & & $\times$ \\
\hline CSUE & & & & & $\times$ & & & & & \\
\hline CACC & $\times$ & $\times$ & $\times$ & & $\times$ & & $\times$ & & & $\times$ \\
\hline CDAT & $\times$ & $\times$ & $\times$ & & $\times$ & & & & & $\times$ \\
\hline CGEN & $\times$ & $\times$ & $\times$ & & $\times$ & & $\times$ & & & $\times$ \\
\hline CPRE & $\times$ & & & & & $\times$ & $\times$ & $\times$ & & $\times$ \\
\hline CSIT & $\times$ & & & & & $\times$ & & $\times$ & & $\times$ \\
\hline CDIR & $\times$ & & & & & $\times$ & & $\times$ & & $\times$ \\
\hline CEXP & $\times$ & $\times$ & $\times$ & & & $\times$ & $\times$ & & & $\times$ \\
\hline CNOU & $\times$ & $\times$ & $\times$ & & $\times$ & & & & & $\times$ \\
\hline CADJ & $\times$ & & & $\times$ & & & $\times$ & $\times$ & $\times$ & $\times$ \\
\hline CVRB & $\times$ & & & & & & & & & $\times$ \\
\hline CSBJ & & & & & & & $\times$ & & & $\times$ \\
\hline CPAR & & & & & & & & & $\times$ & $\times$ \\
\hline CCOM & & & & & & & & & & \\
\hline CREF & & & & & $\times$ & & & & & \\
\hline CPRX & & & & & & $\times$ & & & & \\
\hline \hline AVRB & $\times$ & & & & & & & & & $\times$ \\
\hline ANOU & & $\times$ & & & & & & & & $\times$ \\
\hline ADET & & & & & & & & & & \\
\hline AADJ & & & & $\times$ & & & & & & $\times$ \\
\hline APRE & & & & & & $\times$ & & & & $\times$ \\
\hline ASBJ & & & & & & & $\times$ & & & $\times$ \\
\hline AADV & & & & & & & & $\times$ & & $\times$ \\
\hline APAR & & & & & & & & & $\times$ & $\times$ \\
\hline AAPP & & & & & & & & & \\
\hline
\end{tabular}

CSUB/Verb

Wer jetzt nicht hier ist, wird auch nachher nicht hier sein. 
CSUB/Noun

CSUB/Determiner

Meine Schwester fährt nach Lübeck.

(Deine Schwester wohnt in Berlin und) meine wohnt in Kiel.

CSUB/Pronoun

Das gefält mir nicht.

CSUB/Subjunction

Daß es dir nicht gut geht, tut mir sehr leid.

CSUE/Pronoun ONLY es.

- (i) THERE IS NO OTHER WORD IN FRONT OF THE FINTTE VERB IN A DECLARATTVE SENTENCE:

Es sagt niemand die Wahrheit.

- (ii) es IS AN OBLIGATORY COMPLEMENT:

Es regnet.

$\mathrm{daB}$ es regnet.

- (iii) es IS AN OPTIONAL COMPLEMENT:

Davor ekelt es mich.

- ONLY A FINTTE VERB CAN GOVERN AN EXPLETIVUM (A KIND OF VALENCY

TRANSFORMATION TAKES PLACE; CF. 3.3.):

Es hat niemand über diesen Witz gelacht.

- IN THE CASE OF A VERBAL COMPLEX IN SEVERAL PARTS, THE CAPABILITY TO GOVERN

"CSUE" MOVES TO THE FINITE VERB:

CACC/Verb

Es hat geregnet.

CACC/Noun

Was ich nicht selbst gesehen habe, glaube ich nicht.

CACC/Determiner

Er gab mir meinen Schlüssel wieder.

CACC/Pronoun

Er schenkte mir dieses (und dir jenes Buch).

CACC/Subjunction

Das braucht Peter nicht mehr.

CDAT/Verb

Daß sich die Erde um die Sonne dreht, glaube ich nicht.

CDAT/Noun

CDAT/Determiner

Wem nicht zu vertrauen ist, leiht Peter nichts.

Man hinterlegte dem Gast eine Nachricht.

Mir fehlten zwei (und dir drei Punkte).

CDAT/Pronoun

Mir kommen die Tränen.

CGEN/Verb

Sie entsann sich, wessen sie sich nicht entsinnen wollte.

CGEN/Noun

CGEN/Determiner

Sie entsann sich ihrer ersten Begegnung.

CGEN/Pronoun

Sie entsann sich ihrer ersten (und leider auch ihrer zweiten Begegnung).

CGEN/Subjunction

Man bediente sich seiner.

CPRE/Verb

CPRE/Preposition

Sie entsann sich, daß sie sich kein drittes Mal getroffen hatten.

Worüber sich alle ärgern, ärgere auch ich mich.

Alle setzen auf diesen Mann. 
CPRE/Subjunction

Sie erinnerte sich, daß sie ihn kannte.

CPRE/Adverb

Sie erinnerte sich daran.

CSIT/Verb

Wo Peter wohnt, weiß niemand.

CSIT/Preposition

Er fand einen Arbeitsplatz in einer Maschinenfabrik.

CSIT/Adverb

Das Gesuchte befand sich nicht dort.

CDIR/Verb

Er ging, wohin man ihn auch immer schickte.

CDIR/Preposition

Peter fiel in einen Graben.

CDIR/Adverb

Peter fiel hinein.

CEXP/Verb

Solange die Römer herrschten, war Ruhe in der Provinz.

CEXP/Noun

Er lief zehn Kilometer.

CEXP/Determiner

CEXP/Preposition

Peter lief zehn (und Katrin zwölf Kilometer).

Sie verfehlten sich um eine halbe Stunde.

CEXP/Subjunction

Sie spielten, bis der Morgen graute.

CNOU/Verb

CNOU/Determiner

Er wurde, was er immer werden wollte.

Dieser Mann ist einer, der immer schnell aufgibt.

CNOU/Noun

Er wurde Politiker.

CNOU/Pronoun

CADJ/Verb

Er blieb es auch sein ganzes Leben lang.

CADJ/Adjective

Er benahm sich, wie es nicht anders zu erwarten war.

CADJ/Subjunction

Peter ist krank.

CADJ/Adverb ONLY so

Sie tut, als ob sie nichts verstanden hätte.

Peter ist so.

CADJ/Particle

Peter benimmt sich wie ein Flegel.

Wir sind quitt.

CVRB/Verb

Ich bin es leid.

Peter läßt sich nicht hintergehen.

Ich möchte Klavier spielen können.

CSBJ/Subjunction

Ich möchte Klavier spielen können.

Peter vergaß, uns zu benachrichtigen.

Man vermutet, daß der Täter um zehn Uhr wieder fort war.

Peter scheint verhindert worden zu sein. 
CPAR/Particle ONLY als

Das Buch gilt als Bestseller.

Das Buch gilt als lesbar.

Man sah ihn als einen offiziellen Teilnehmer an.

CREF/Pronoun ONLY REFLEXIVE PRONOUNS COREFERENTIAL WITH THE SUBJECT:

Er verhielt sich sehr sonderbar.

CPRX/Preposition VERBS WITH PREFIXOIDE (NON-FIXED PREFI), WHICH OCCUR IN THE FIRST OR SECOND POSITION:

Das Fest findet auch bei Regen statt.

AVRB/Verb

Sie verließ uns plötzlich, was uns alle sehr erstaunte.

Sie verpackten die Geschenke, wie sie es immer taten.

ANOU/Noun

Maria stand eines Tages vor der Tür.

Das ist meines Erachtens falsch.

Nächsten Dienstag heiraten sie.

AADJ/Adjective

Man verpackte vergnügt die Geschenke.

Man verpackte umständlich die Geschenke.

APRE/Preposition

In drei Stunden kommt Peter.

Mit Ihnen trinke ich am liebsten.

ASBJ/Subjunction

Als Peter zehn Jahre alt war, zog seine Familie ins Ausland.

Georg trainiert, um Kondition zu bekommen.

AADV/Adverb

Dann tranken sie Kaffee.

Peter fährt lieber mit Katrin.

APAR/Particle

Dieses Argument ist allerdings recht überzeugend.

Er wird wohl kommen.

\section{Correlates}

All Vs, which govern a dependent of type X expressed as a VP (with the exception of CVRB), can optionally govern another dependent $\mathrm{X}^{\prime}$, which is always expressed by a Pro or an Adv. A dependent of this kind is called a correlate.

The possible correlates are:

CACC: es, das

CDAT: dem, diesem or jenem

CGEN: dessen

CPRE: prepositional adverb

CSIT: das

CDIR: dahin, daher

CEXP: es

CNOU: das

CADJ: es

CSBJ: $e s, d a s$ (only with $d a \beta$ or $o b$ as the head of CSBJ)

CSUB'/Pronoun

CACC'/Pronoun

Wer jetzt nicht hier ist, der wird auch nachher nicht hier sein.

Was ich nicht selbst gesehen habe, das glaube ich auch nicht. 
CDAT'/Pronoun

Wem nicht zu vertrauen ist, dem leiht Peter nichts.

CGEN'/Pronoun

Wessen sie sich nicht entsinnen wollte, dessen entsann sie sich doch.

CPRE'/Adverb

Worüber sich alle ärgern, darüber ärgere auch ich mich.

CSIT'/Pronoun

Das weiß niemand, wo Peter wohnt.

CDIR'/Adverb

Er ging dahin, wohin man ihn auch immer schickte.

CNOU'/Pronoun

Er wurde das, was er immer werden wollte.

CADJ'/Adverb

Er benahm sich so, wie es nicht anders zu erwarten war.

CSBJ'Pronoun

Peter vergaß es, uns zu benachrichtigen.

\subsection{Dependents of nouns}

\begin{tabular}{|l|l|l|l|l|l|l|l|l|c|c|}
\hline & $\mathbf{V}$ & $\mathbf{N}$ & Det & A & Pro & P & Sbj & Adv & Par & Con \\
\hline \hline CSUB & & & & & & & & & & \\
\hline CSUE & & & & & & & & & & \\
\hline CACC & & & & & & & & & & \\
\hline CDAT & & & & & & & & & & \\
\hline CGEN & & & & & & & & & & \\
\hline CPRE & & & & & & $\times$ & & $\times$ & & $\times$ \\
\hline CSIT & & & & & & & & & & \\
\hline CDIR & & & & & & $\times$ & & $\times$ & & $\times$ \\
\hline CEXP & & $\times$ & $\times$ & & & $\times$ & & & & $\times$ \\
\hline CNOU & & $\times$ & & & & & & & & \\
\hline CADJ & & & & & & & & & & \\
\hline CVRB & & & & & & & & & & \\
\hline CSBJ & & & & & & & $\times$ & & & $\times$ \\
\hline CPAR & & & & & & & & & & \\
\hline CCOM & & & & & & & $\times$ & $\times$ & $\times$ & $\times$ \\
\hline CREF & & & & & & $\times$ & & & & \\
\hline CPRX & & & & & & & & & & \\
\hline \hline AVRB & $\times$ & & & & & & & & & $\times$ \\
\hline ANOU & & $\times$ & $\times$ & & & & & & & $\times$ \\
\hline ADET & & & $\times$ & & & & & & & $\times$ \\
\hline AADJ & & & & $\times$ & & & & & & $\times$ \\
\hline APRE & & & & & $\times$ & & & & $\times$ \\
\hline ASBJ & & & & & & & & & & \\
\hline AADV & & & & & & & & $\times$ & & $\times$ \\
\hline APAR & & & & & & & & $\times$ & $\times$ \\
\hline AAPP & & $\times$ & $\times$ & $\times$ & & $\times$ & & & & $\times$ \\
\hline
\end{tabular}


CPRP/Preposition

die Hoffnung auf Frieden

der Gedanke an Japan

CPRP/Adverb

CDIR/Preposition

die Hoffnung darauf

CDIR/Adverb

die Fahrt in die Berge

CEXP/Noun

CEXP/Determiner

die Fahrt dahin

das Laufen eines halben Kilometers

CEXP/Preposition

das Laufen eines halben (und das Gehen eines ganzen Kilometers)

CNOU/Noun

eine Theateraufführung von einer Stunde

Bundeskanzler Kohl

ein Glas warme Milch

CSBJ/Subjunction

der Gedanke, daß Hans bald kommt,

CCOM/Subjunction

eine Qualität, wie sie ihresgleichen sucht,

CCOM/Adverb ONLY so

so eine Qualität

CCOM/Particle

das Größersein als zwei Meter

CREF/Pronoun

das sich Weigern

AVRB/Verb

der Mann, der Birnen verkauft,

ANOU/Noun

das Haus meines Vaters

das Laufen der Kinder

ANOU/Determiner

das Haus meines (und der Garten deines Vaters)

das Laufen der vier (und das Schwimmen der fünf Schulkinder)

ADET/Determiner

der Mann

dieser Mann

alle Männer

AADJ/Adjective

zwanzig Männer

der hohe Berg

meine wenigen Kenntnisse

APRE/Preposition

der laufende Junge

AADV/Adverb

der höchste Berg in dieser Gegend

der Mann dort

APAR/Particle

mein Vater als Schüler

vielleicht mein Vater

AAPP/Noun

Herr Meier, Vorsitzender dieses Vereins, 
AAPP/Adjective

AAPP/Preposition

Friederike, wie immer vergnügt,

ihre Freundin, in bester Laune,

\subsection{Dependents of determiners}

The dependents of Det are in general identical with the dependents of N. DetPs result from noun phrases in which an ellipsis of the head has taken place. For that reason, the dependents of Det are the same as those of $\mathrm{N}$. The dependency representation itself makes no use of ellipsis.

\begin{tabular}{|l|l|l|l|l|l|l|l|l|l|c|}
\hline & $\mathbf{V}$ & $\mathbf{N}$ & Det & A & Pro & P & Sbj & Adv & Par & Con \\
\hline \hline CSUB & & & & & & & & & & \\
\hline CSUE & & & & & & & & & & \\
\hline CACC & & & & & & & & & & \\
\hline CDAT & & & & & & & & & & \\
\hline CGEN & & & & & & & & & & \\
\hline CPRE & & & & & & $\times$ & & $\times$ & & $\times$ \\
\hline CSIT & & & & & & & & & & \\
\hline CDIR & & & & & & $\times$ & & $\times$ & & $\times$ \\
\hline CEXP & & $\times$ & $\times$ & & & $\times$ & & & & $\times$ \\
\hline CNOU & & & & & & & & & & \\
\hline CADJ & & & & & & & & & & \\
\hline CVRB & & & & & & & & & & \\
\hline CSBJ & & & & & & & $\times$ & & & $\times$ \\
\hline CPAR & & & & & & & & & & \\
\hline CCOM & & & & & & & $\times$ & $\times$ & $\times$ & $\times$ \\
\hline CREF & & & & & & $\times$ & & & & \\
\hline CPRX & & & & & & & & & & \\
\hline \hline AVRB & $\times$ & & & & & & & & & $\times$ \\
\hline ANOU & & $\times$ & $\times$ & & & & & & & $\times$ \\
\hline ADET & & & $\times$ & & & & & & & $\times$ \\
\hline AADJ & & & & $\times$ & & & & & & $\times$ \\
\hline APRE & & & & & & $\times$ & & & & $\times$ \\
\hline ASBJ & & & & & & & & & & \\
\hline AADV & & & & & & & & $\times$ & & $\times$ \\
\hline APAR & & & & & & & & & $\times$ & $\times$ \\
\hline AAPP & & $\times$ & $\times$ & $\times$ & & $\times$ & & & & $\times$ \\
\hline
\end{tabular}

CPRP/Preposition

(die Hoffnung auf Gerechtigkeit und) die auf Frieden

(der Gedanke an China und) der an Japan

CPRP/Adverb

(meine Hoffnung auf Frieden und) deine darauf

CDIR/Preposition

(unsere Fahrt an die See und) eure in die Berge

CDIR/Adverb

(unsere Fahrt in die Berge und) eure dahin 


\section{CEXP/Noun}

CEXP/Determiner

(das Laufen eines ganzen und) das eines halben Kilometers

(das Laufen eines ganzen Kilometers und) das eines halben

CEXP/Preposition

(eine Aufführung von zwei und) eine von einer Stunde

CSBJ/Subjunction

(der Gedanke an Katrin und) der, daß Hans bald kommt,

etc. like $\mathbf{N}$

\subsection{Dependents of adjectives}

\begin{tabular}{|c|c|c|c|c|c|c|c|c|c|c|}
\hline & $\mathbf{V}$ & $\mathbf{N}$ & Det & A & Pro & P & Sbj & Adv & Par & Con \\
\hline \hline CSUB & & & & & & & & & & \\
\hline CSUE & & & & & & & & & & \\
\hline CACC & & $\times$ & & & $\times$ & & & & & $\times$ \\
\hline CDAT & & $\times$ & & & $\times$ & & & & & $\times$ \\
\hline CGEN & & $\times$ & & & $\times$ & & & & & $\times$ \\
\hline CPRE & & & & & & $\times$ & & $\times$ & & $\times$ \\
\hline CSIT & & & & & & $\times$ & & $\times$ & & $\times$ \\
\hline CDIR & & & & & & $\times$ & & $\times$ & & $\times$ \\
\hline CEXP & & $\times$ & & & & $\times$ & & $\times$ & & $\times$ \\
\hline CNOU & & $\times$ & $\times$ & & $\times$ & & & & & $\times$ \\
\hline CADJ & & & $\times$ & & & $\times$ & $\times$ & $\times$ & $\times$ \\
\hline CVRB & $\times$ & & & & & & & & & $\times$ \\
\hline CSBJ & & & & & & & $\times$ & & & $\times$ \\
\hline CPAR & & & & & & & & & $\times$ & $\times$ \\
\hline CCOM & & & & & & $\times$ & $\times$ & $\times$ & $\times$ \\
\hline CREF & & & & & $\times$ & & & & & \\
\hline CPRX & & & & & & & & & & \\
\hline \hline AVRB & & & & & & & & & & \\
\hline ANOU & & & & & & & & & & \\
\hline ADET & & & & & & & & & & \\
\hline AADJ & & & & & & & & & & \\
\hline APRE & & & & & $\times$ & & & & $\times$ \\
\hline ASBJ & & & & & & & $\times$ & & & $\times$ \\
\hline AADV & & & & & & & & & & \\
\hline APAR & & & & & & & & & & \\
\hline AAPP & & & & & & & & $\times$ & $\times$ \\
\hline
\end{tabular}

CACC/Noun MAINLY ADJECTIVAL PARTICIPLES:

CACC/Pronoun

der seine Brille suchende Peter

der dieses suchende Peter

CDAT/Noun

ein ihrer Familie nahestehender Bekannter

CDAT/Pronoun

der ihm etwas Geld gebende Mann

CGEN/Noun

ein sich seines Erfolges sicherer Mann 


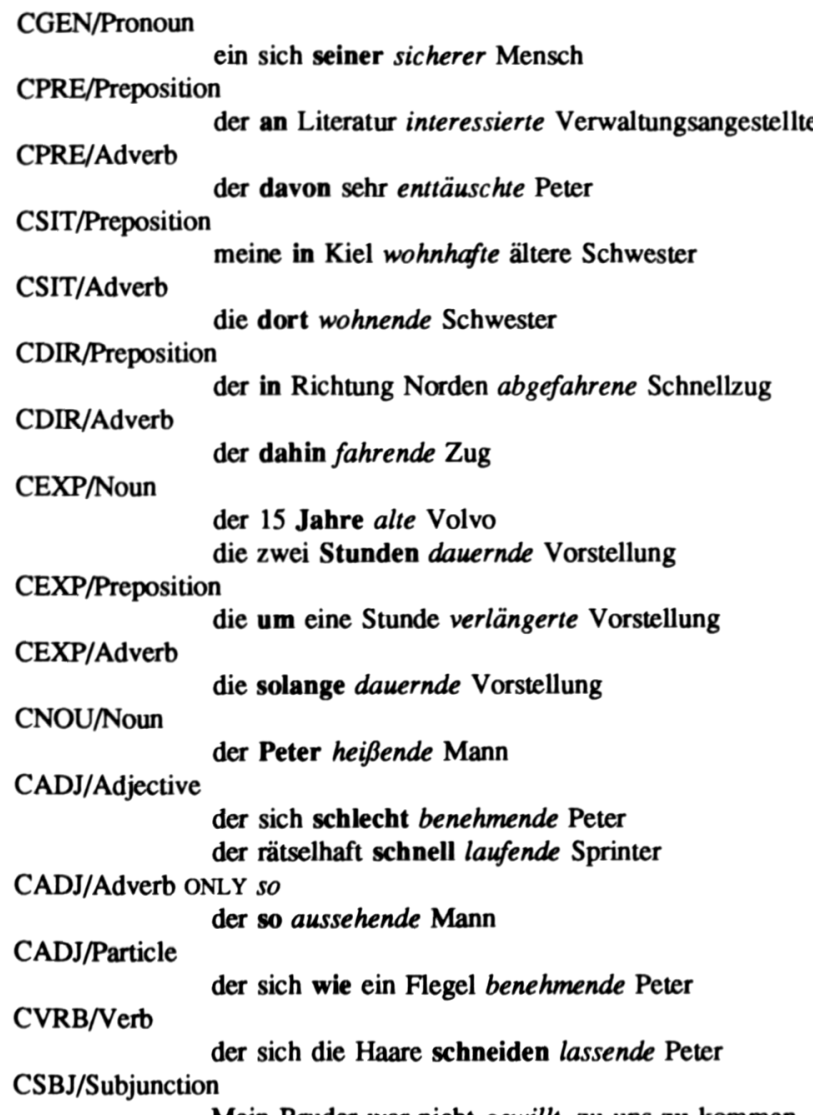

Mein Bruder war nicht gewillt, zu uns zu kommen.

ein solches Unwetter, daß man nicht hinausgehen konnte,

CPAR/Particle

der als Betrüger geltende Bankier

zu schön, um wahr zu sein,

CCOM/Subjunction

ein sehr erfreuliches Ereignis

CCOM/Adverb ONLY so

besser, als wir erwartet hatten,

CCOM/Particle

so gut wie im letzten Jahr

besser als im letzten Jahr

CREF/Pronoun ONLY sich

der sich schlecht benehmende Peter

APRE/Preposition

ein meiner Meinung nach unverdientes Lob

ASBJ/Subjunction

die in ihrem blauen Kleid weggehende Katrin

alt, wie das Haus nun einmal war,

wohnhaft in Kiel, wie sie wahrheitsgetreu angegeben hat, 
APAR/Particle

ein wohl recht neues Auto

eine möglicherweise ungerechte Entscheidung

\subsection{Dependents of pronouns}

\begin{tabular}{|c|c|c|c|c|c|c|c|c|c|c|}
\hline & $\mathrm{V}$ & $\mathbf{N}$ & Det & $\mathbf{A}$ & Pro & $\mathrm{P}$ & Sbj & Adv & Par & Con \\
\hline \multicolumn{11}{|l|}{ CSUB } \\
\hline \multicolumn{11}{|l|}{ CSUE } \\
\hline \multicolumn{11}{|l|}{ CACC } \\
\hline \multicolumn{11}{|l|}{ CDAT } \\
\hline \multicolumn{11}{|l|}{ CGEN } \\
\hline CPRE & & & & & & $x$ & & $x$ & & $x$ \\
\hline \multicolumn{11}{|l|}{ CSIT } \\
\hline \multicolumn{11}{|l|}{ CDIR } \\
\hline \multicolumn{11}{|l|}{ CEXP } \\
\hline CNOU & & $x$ & & $x$ & & & & & & $x$ \\
\hline CADJ & & & & $x$ & & & $x$ & & $x$ & $x$ \\
\hline CVRB & $x$ & & & & & & & & & $x$ \\
\hline \multicolumn{11}{|l|}{ CSBJ } \\
\hline CPAR & & & & & & & & & $x$ & $x$ \\
\hline \multicolumn{11}{|l|}{ CCOM } \\
\hline \multicolumn{11}{|l|}{ CREF } \\
\hline \multicolumn{11}{|l|}{ CPRX } \\
\hline \multicolumn{11}{|l|}{ AVRB } \\
\hline \multicolumn{11}{|l|}{ ANOU } \\
\hline \multicolumn{11}{|l|}{ ADET } \\
\hline \multicolumn{11}{|l|}{ AADJ } \\
\hline \multicolumn{11}{|l|}{ APRE } \\
\hline \multicolumn{11}{|l|}{ ASBJ } \\
\hline \multicolumn{11}{|l|}{ AADV } \\
\hline \multicolumn{11}{|l|}{ APAR } \\
\hline AAPP & & & & & & & & & & \\
\hline
\end{tabular}

CPRE/Preposition

$d u$ mit deinem Glück

etwas von der Mettwurst

$e r$, im Vollbesitz seiner Kräfte,

CPRE/Adverb

$d u$ dort

CNOU/Noun

$d u$ Idiot

ich, ein aufrechter Demokrat,

CNOU/Adjective

CADJ/Adjective

$e r$, schon nicht mehr ganz nüchtern,

nichts Gutes 
CADJ/Subjunction

nichts, wie es sein soll,

CADJ/Particle

nichts wie bei uns

CVRB/Verb

$d u$, der du mir geholfen hast,

nichts, was mir helfen könnte,

CPAR/Particle

er als Mathematiker

4.6. Dependents of prepositions

\begin{tabular}{|c|c|c|c|c|c|c|c|c|c|c|}
\hline & $\mathrm{V}$ & $\mathbf{N}$ & Det & $\mathbf{A}$ & Pro & $\mathrm{P}$ & Sbj & Adv & Par & Con \\
\hline \multicolumn{11}{|l|}{ CSUB } \\
\hline \multicolumn{11}{|l|}{ CSUE } \\
\hline CACC & & $x$ & $x$ & & $x$ & & & $x$ & & $x$ \\
\hline CDAT & & $x$ & $x$ & & $x$ & & & & & $x$ \\
\hline CGEN & & $x$ & $x$ & & $x$ & & & & & $x$ \\
\hline \multicolumn{11}{|l|}{ CPRE } \\
\hline \multicolumn{11}{|l|}{ CSIT } \\
\hline \multicolumn{11}{|l|}{ CDIR } \\
\hline \multicolumn{11}{|l|}{ CEXP } \\
\hline \multicolumn{11}{|l|}{$\mathrm{CNOU}$} \\
\hline \multicolumn{11}{|l|}{ CADJ } \\
\hline \multicolumn{11}{|l|}{ CVRB } \\
\hline \multicolumn{11}{|l|}{ CSBJ } \\
\hline CPAR & & & & & & & & $x$ & & $x$ \\
\hline \multicolumn{11}{|l|}{ CCOM } \\
\hline \multicolumn{11}{|l|}{ CREF } \\
\hline \multicolumn{11}{|l|}{ CPRX } \\
\hline \multicolumn{11}{|l|}{ "AVRB } \\
\hline \multicolumn{11}{|l|}{ ANOU } \\
\hline \multicolumn{11}{|l|}{ ADET } \\
\hline \multicolumn{11}{|l|}{ AADJ } \\
\hline \multicolumn{11}{|l|}{ APRE } \\
\hline \multicolumn{11}{|l|}{ ASBJ } \\
\hline \multicolumn{11}{|l|}{ AADV } \\
\hline \multicolumn{11}{|l|}{ APAR } \\
\hline AAPP & & & & & & & & & & \\
\hline
\end{tabular}

CACC/Noun

für deinen Freund

in die Grube

CACC/Determiner

für deinen (, nicht für meinen Freund)

gegen fünf

um sieben

CACC/Pronoun

für ihn 
CDAT/Noun

mit der Straßenbahn

aus diesem Grund

CDAT/Determiner

mit dieser (, nicht mit jener Straßenbahn)

CDAT/Pronoun

mit niemandem

CGEN/Noun

wegen des Geldes

CGEN/Determiner

wegen deiner (, nicht wegen meiner Erkrankung)

CGEN/Pronoun

CPAR/Adverb

wegen ihr

nach unten

bis jetzt

\subsection{Dependents of subjunctions}

\begin{tabular}{|c|c|c|c|c|c|c|c|c|c|c|}
\hline & $\mathbf{V}$ & $\mathbf{N}$ & Det & A & Pro & $P$ & Sbj & Adv & Par & Con \\
\hline \multicolumn{11}{|l|}{ CSUB } \\
\hline \multicolumn{11}{|l|}{ CSUE } \\
\hline \multicolumn{11}{|l|}{ CACC } \\
\hline \multicolumn{11}{|l|}{ CDAT } \\
\hline \multicolumn{11}{|l|}{ CGEN } \\
\hline \multicolumn{11}{|l|}{ CPRE } \\
\hline \multicolumn{11}{|l|}{ CSIT } \\
\hline \multicolumn{11}{|l|}{ CDIR } \\
\hline \multicolumn{11}{|l|}{ CEXP } \\
\hline \multicolumn{11}{|l|}{ CNOU } \\
\hline CADJ & & & & $x$ & & & & & & $x$ \\
\hline CVRB & $x$ & & & & & & & & & $x$ \\
\hline CSBJ & & & & & & & $x$ & & & $x$ \\
\hline \multicolumn{11}{|l|}{ CPAR } \\
\hline \multicolumn{11}{|l|}{ CCOM } \\
\hline \multicolumn{11}{|l|}{ CREF } \\
\hline \multicolumn{11}{|l|}{ CPRX } \\
\hline \multicolumn{11}{|l|}{ AVRB } \\
\hline \multicolumn{11}{|l|}{ ANOU } \\
\hline \multicolumn{11}{|l|}{ ADET } \\
\hline \multicolumn{11}{|l|}{ AADJ } \\
\hline \multicolumn{11}{|l|}{ APRE } \\
\hline \multicolumn{11}{|l|}{ ASBJ } \\
\hline \multicolumn{11}{|l|}{ AADV } \\
\hline \multicolumn{11}{|l|}{ APAR } \\
\hline AAPP & & & & & & & & & & \\
\hline
\end{tabular}

CADJ/Adjective

falls vom Arzt nicht anders verordnet obwohl noch sehr jung 
CVRB/Verb

nachdem alle gekommen waren

$d a \beta$ sie nicht hier ist

CSBJ/Subjunction

Er ist schon zu weit entfernt, als daß wir ihn noch einholen können.

Es sieht aus, als ob es gebrannt hat.

Er verließ uns, ohne sich von uns verabschiedet zu haben.

\subsection{Dependents of adverbs}

\begin{tabular}{|c|c|c|c|c|c|c|c|c|c|c|}
\hline & $\mathrm{V}$ & $\mathbf{N}$ & Det & $\mathbf{A}$ & Pro & $\mathbf{P}$ & Sbj & Adv & Par & Con \\
\hline \multicolumn{11}{|l|}{ CSUB } \\
\hline \multicolumn{11}{|l|}{ CSUE } \\
\hline \multicolumn{11}{|l|}{ CACC } \\
\hline \multicolumn{11}{|l|}{ CDAT } \\
\hline \multicolumn{11}{|l|}{ CGEN } \\
\hline \multicolumn{11}{|l|}{ CPRE } \\
\hline \multicolumn{11}{|l|}{ CSIT } \\
\hline \multicolumn{11}{|l|}{ CDIR } \\
\hline \multicolumn{11}{|l|}{ CEXP } \\
\hline \multicolumn{11}{|l|}{$\mathrm{CNOU}$} \\
\hline \multicolumn{11}{|l|}{ CADJ } \\
\hline \multicolumn{11}{|l|}{ CVRB } \\
\hline \multicolumn{11}{|l|}{ CSBJ } \\
\hline \multicolumn{11}{|l|}{ CPAR } \\
\hline CCOM & & & & & & & $x$ & $x$ & $x$ & $x$ \\
\hline \multicolumn{11}{|l|}{ CREF } \\
\hline \multicolumn{11}{|l|}{ CPRX } \\
\hline \multicolumn{11}{|l|}{ AVRB } \\
\hline \multicolumn{11}{|l|}{ ANOU } \\
\hline \multicolumn{11}{|l|}{ ADET } \\
\hline \multicolumn{11}{|l|}{ AADJ } \\
\hline \multicolumn{11}{|l|}{ APRE } \\
\hline ASBJ & & & & & & & $x$ & & & $x$ \\
\hline AADV & & & & & & & & $x$ & & $x$ \\
\hline APAR & & & & & & & & & $x$ & $x$ \\
\hline AAPP & & & & & & & & & & \\
\hline
\end{tabular}

$\mathrm{CCOM} /$ Subjunction

eher, als wir erwartet hatten,

CCOM/Adverb ONLY so

so gern wie erwartet

CCOM/Particle

eher als Peter

ASBJ/Subjunction

AADV/Adverb

gestern, als ich spazierenging,

immer hier 
APAR/Particle

möglicherweise hier

ganz hinten

\subsection{Dependents of particles}

\begin{tabular}{|c|c|c|c|c|c|c|c|c|c|c|}
\hline & $\mathrm{V}$ & $\mathbf{N}$ & Det & À & Pro & $P$ & Sbj & Adv & Par & Con \\
\hline \multicolumn{11}{|l|}{ CSUB } \\
\hline \multicolumn{11}{|l|}{ CSUE } \\
\hline \multicolumn{11}{|l|}{ CACC } \\
\hline \multicolumn{11}{|l|}{ CDAT } \\
\hline \multicolumn{11}{|l|}{ CGEN } \\
\hline CPRE & & & & & & $x$ & & $x$ & & $x$ \\
\hline \multicolumn{11}{|l|}{ CSIT } \\
\hline \multicolumn{11}{|l|}{ CDIR } \\
\hline \multicolumn{11}{|l|}{ CEXP } \\
\hline CNOU & & $x$ & $x$ & & $x$ & & & & & $x$ \\
\hline CADJ & & & & $x$ & & & & & & $x$ \\
\hline \multicolumn{11}{|l|}{ CVRB } \\
\hline CSBJ & & & & & & & $x$ & & & $x$ \\
\hline \multicolumn{11}{|l|}{ CPAR } \\
\hline \multicolumn{11}{|l|}{ CCOM } \\
\hline \multicolumn{11}{|l|}{ CREF } \\
\hline \multicolumn{11}{|l|}{ CPRX } \\
\hline \multicolumn{11}{|l|}{ AVRB } \\
\hline \multicolumn{11}{|l|}{ ANOU } \\
\hline \multicolumn{11}{|l|}{ ADET } \\
\hline \multicolumn{11}{|l|}{ AADJ } \\
\hline \multicolumn{11}{|l|}{ APRE } \\
\hline \multicolumn{11}{|l|}{ ASBJ } \\
\hline \multicolumn{11}{|l|}{ AADV } \\
\hline \multicolumn{11}{|l|}{ APAR } \\
\hline AAPP & & & & & & & & & & \\
\hline
\end{tabular}

\section{CPRE/Preposition}

schöner als in dieser Gegend

CPRE/Adverb

so gern wie im letzten Jahr

schöner als dort

so schön wie hier

$z u$ jung dafür

CNOU/Noun

so groß wie mein Freund Peter

lieber als diese Leute

CNOU/Determiner

kleiner als mein (, aber größer als dein Freund)

CNOU/Pronoun

so groß wie du 
CADJ/Adjective

Das Buch gilt als lesbar.

Er sieht aus wie betrunken.

CSBJ/Subjunction

$z u$ schön, um wahr zu sein,

Sie scheint eher zu lachen als zu weinen.

\subsection{Coordination}

Conjunctions always have the capability to double a dependency relation. For instance, if a word can govern a dependent of the CACC type, the conjunction und can govern two dependents of the same type, i.e. und $\angle \mathrm{CACC}, \mathrm{CACC}\rangle$. The default valency of all conjunctions is $<$ CVRB, CVRB $>$. Sample sentences with governors from various word classes:

CACC/Conjunction

CADJ/Conjunction

Er gab mir den Schlüssel und das Buch wieder.

Hans benimmt sich manchmal gut und manchmal schlecht.

CPRE/Conjunction

ADET/Conjunction

die Hoffnung auf Frieden und auf Gerechtigkeit

mein und dein Buch

AAPP/Conjunction

Friederike, wie immer vergnügt und in bester Laune,

CDIR/Conjunction

CPAR/Conjunction

(eure Fahrt in die Berge und) unsere nach Italien oder nach Spanien.

CPRE/Conjunction

der als Betrüger und als der Kopf der Bande geltende Mann

etwas von der Mettwurst und von der Salami

CGEN/Conjunction

CVRB/Conjunction

wegen ihr oder ihres Geldes

nachdem alle angekommen waren und sich gesetzt hatten

CSBJ/Conjunction

gestern, als ich spazierenging und darüber nachdachte,

CADJ/Conjunction

das Buch gilt als lehrreich und spannend.

A coordinate stucture behaves like a single word for elements which are not in one of the dependent phrases. That means that the capability of the heads of both coordinated phrases to govern certain dependents has moved to the conjunction. If there is, for instance, a structure involving coordination of two nouns, the conjunction can govern all the dependents which can also be governed by the nouns: 
dein Freund und Helfer

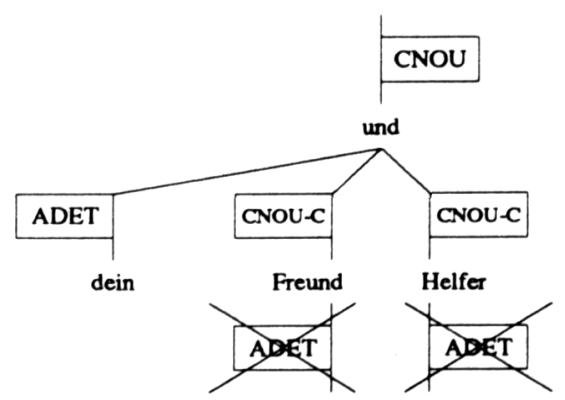

CSUB/Pronoun WHERE "CON" GOVERNS FINTIE VS

Sie lacht und weint gleichzeitig.

ADET/Determiner WHERE "CON" GOVERNS NS

der Bundeskanzler und Parteivorsitzende

CPAR/Particle WHERE "CON" GOVERNS AS

sehr groB und stark

etc.

\section{Sample sentence}

Nachdem Müller, gerade aus Berlin, wo er für seine Sache erfolgreich geworben hatte, zurückgekehrt, als erster geredet hatte und sich setzte, erhob sich frenetischer Beifall, anstatt daß die Leute schwiegen, und niemand wußte, was das zu bedeuten hatte.

'After Müller, having just come back from Berlin, where he had successfully fought for his case, had been the first to speak and sat down, there was frenzied applause instead of silence, and no-one knew what it might mean.' 


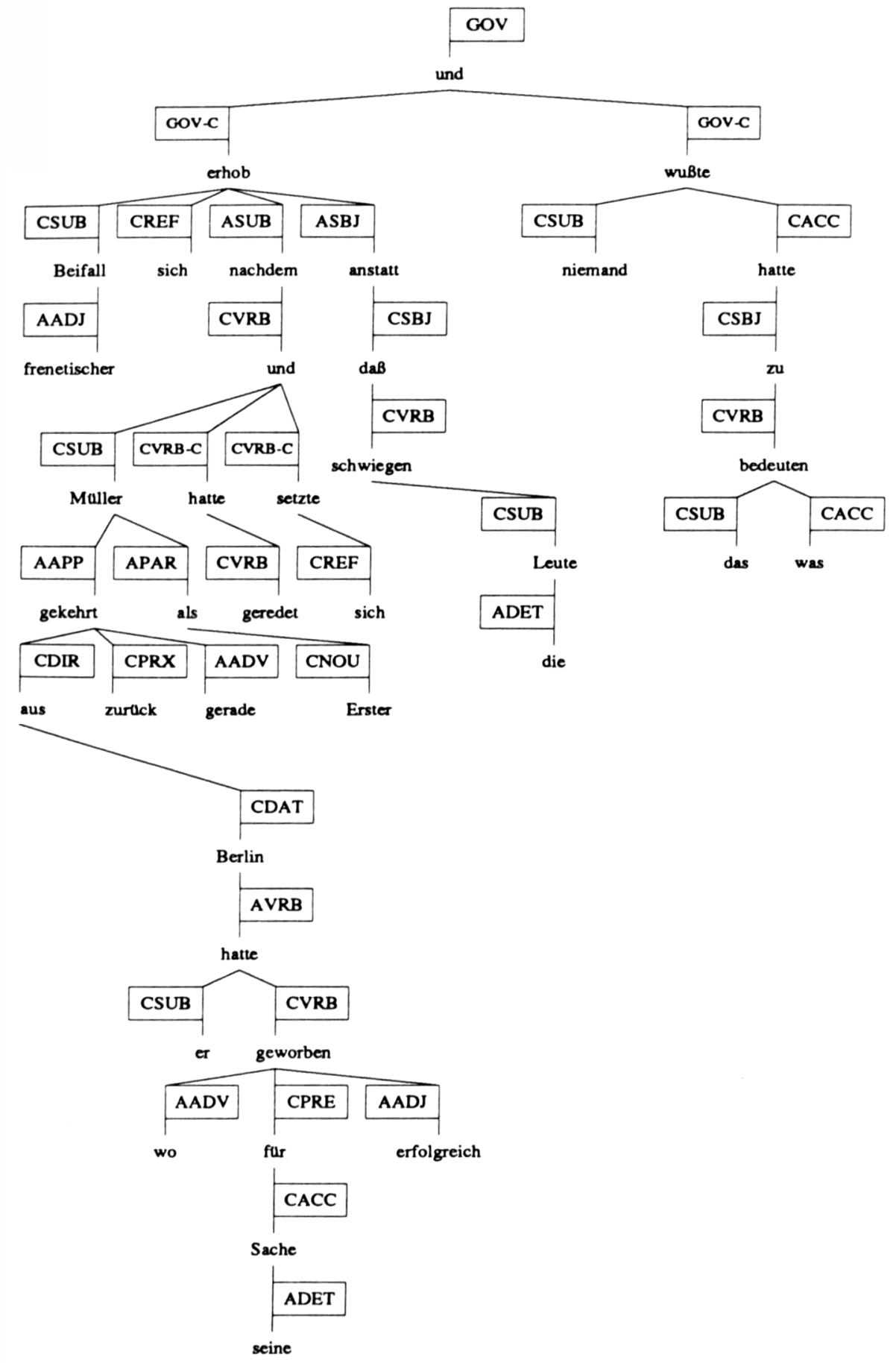




\section{Note}

I am grateful to Prof. Ulrich Engel (Institut für Deutsche Sprache, Mannheim) for his valuable comments on a former version of this syntax. My work is by and large based on his studies of German grammar (especially Engel 1982, 1988).

\section{References}

Engel, Ulrich (1982): Syntax der deutschen Gegenwartssprache.

Berlin: Schmidt, 2nd rev. ed.

Engel, Ulrich (1988): Deutsche Grammatik.

Heidelberg: Groos 